Proyecciones Journal of Mathematics

Vol. 30, No 2, pp. 137-148, August 2011.

Universidad Católica del Norte

Antofagasta - Chile

\title{
On binomial operator representations of certain polynomial
}

\author{
MUMTAZ AHMAD KHAN \\ and \\ NISAR K. S. \\ ALIGARH MUSLIM UNIVERSITY, INDIA \\ Received: March 2011. Accepted : May 2011
}

\begin{abstract}
Based on the technique used by M. A. Khan and A. K. Shukla [4] here finite series representations of bionomial partial differential operators have been used to establish operator representations of various polynomials not considered in the earlier mentioned paper. The results obtained are believed to be new.
\end{abstract}

AMS (MOS) subject classification (2000) : Primary 47F05, Secondary $33 C 45$.

Keywords : Binomial operator, Operational represenations. 


\section{Introduction :}

Recently in 2009, M. A. Khan and A. K. Shukla [4] evolved a new technique to give operator representations of certain polynomials. They give binomial and trinomial operator representation of certain polynomials. The aim of the presnt paper is to strengthen the technique evolved by obtaining binomial operator represenations of some more polynomials not considered in the above mentioned paper.

\section{The definition, notations and results used :}

In deriving the operational representations of various polynomials use has been made of the fact that

$$
D^{\mu} x^{\lambda}=\frac{\Gamma(1+\lambda)}{\Gamma(1+\lambda-\mu)} x^{\lambda-\mu}, \quad D \equiv \frac{d}{d x}
$$

Where $\lambda$ and $\mu, \lambda \geq \mu$ are arbitrory real numbers.

In particular, use has been made of the following results:

$$
\begin{gathered}
D^{r} e^{-x}=(-1)^{r} e^{-x} \\
D^{r} x^{-\alpha}=(\alpha)_{r}(-1)^{r} x^{-\alpha-r}, \alpha \text { is not an integer } \\
D^{r} x^{-\alpha-n}=(\alpha+n)_{r}(-1)^{r} x^{-\alpha-n-r} \\
D^{n-r} x^{\alpha-1+n}=\frac{(\alpha)_{n}}{(\alpha)_{r}} x^{\alpha-1+r} \\
D^{n-r} x^{-\alpha}=\frac{(\alpha)_{n}(-1)^{n}}{(1-\alpha-n)_{r}} x^{-\alpha-n+r}, \alpha \text { is not an integer }
\end{gathered}
$$

Where $n$ and $r$ are denote positive integrs and 


$$
(\alpha)_{n}=\alpha(\alpha+1) \cdots \cdots(\alpha+n-1) ; \quad(\alpha)_{0}=1
$$

We also need the defenitions of the following polynomials interms of hypergeometric function and also their notations (see [1],[3], [9], [10]).

\section{CESARO POLYNOMIALS}

It is denoted by the symbol $g_{n}^{(s)}(x)$ and is defined as

$$
g_{n}^{(s)}(x)=\left(\begin{array}{c}
s+n \\
n
\end{array}\right){ }_{2} F_{1}\left[\begin{array}{c}
-n, 1 ; x \\
-s-n ; x
\end{array}\right]
$$

\section{SYLVESTER POLYNOMIALS}

It is denoted by the symbol $\varphi_{n}(x)$ and is defined as

$$
\varphi_{n}(x)=\frac{x^{n}}{n !}{ }_{2} F_{0}\left[\begin{array}{rr}
-n, & x ; \\
-; & x^{-1}
\end{array}\right]
$$

\section{SHIVELY'S PSEUDO LAGUERRE POLYNOMIALS}

It is denoted by the symbol $R_{n}(a, x)$ and is defined as

$$
R_{n}(a, x)=\frac{(a)_{2 n}}{n !(a)_{n}}{ }_{1} F_{1}\left[\begin{array}{cc}
-n ; & x \\
a+n ;
\end{array}\right]
$$

\section{HERMITE POLYNOMIAL}

It is denoted by the symbol $H_{n}(x)$ and is defined as

$$
H_{n}(x)=(2 x)^{n}{ }_{2} F_{0}\left[\begin{array}{rr}
-\frac{1}{2} n,-\frac{1}{2} n+\frac{1}{2} ; & \frac{1}{x^{2}}
\end{array}\right]
$$

\section{BEDIENT POLYNOMIALS}

Bedient [2], in his study of some polynomials assosiated with Appell's $F_{2}$ and $F_{3}$ introduced 
(2.11)

$$
R_{n}(\beta, \gamma, x)=\frac{(\beta)_{n}(2 x)^{n}}{n !}{ }_{3} F_{2}\left[\begin{array}{r}
-\frac{n}{2},-\frac{n}{2}+\frac{1}{2}, \gamma-\beta ; \\
\gamma, 1-\beta-n ;
\end{array} \quad \frac{1}{x^{2}}\right]
$$

$$
G_{n}(\alpha, \beta ; x)=\frac{(\alpha)_{n}(\beta)_{n}(2 x)^{n}}{n !(\alpha+\beta)_{n}}{ }_{3} F_{2}\left[\begin{array}{r}
-\frac{1}{2} n,-\frac{1}{2} n+\frac{1}{2}, 1-\alpha-\beta-n ; \\
1-\alpha-n, 1-\beta-n ;
\end{array} \quad \frac{1}{x^{2}}\right]
$$

\section{LOMMEL POLYNOMIAL}

It is denoted by the symbol $R_{n, \nu}\left(\frac{1}{z}\right)$ and is defined as

$$
R_{n, \nu}\left(\frac{1}{z}\right)=(\nu)_{n}(2 z)^{n}{ }_{2} F_{3}\left[\begin{array}{cc}
-\frac{1}{2} n,-\frac{1}{2} n+\frac{1}{2} ; & -\frac{1}{z^{2}} \\
\nu,-n, 1-\nu-n ; & -1
\end{array}\right.
$$

\section{BRAFMAN POLYNOMIAL}

It is denoted by the symbol $B_{n}^{m}\left[\alpha_{1}, \alpha_{2} \cdots \alpha_{r}, \quad \beta_{1}, \beta_{2} \cdots \beta_{s} ; x\right]$ and is defined as

$$
B_{n}^{m}\left[\alpha_{1}, \alpha_{2} \cdot \alpha_{r}, \beta_{1}, \beta_{2} \cdot \beta_{s} ; x\right]={ }_{m+r} F_{s}\left[\begin{array}{r}
\Delta(m ;-n), \alpha_{1}, \alpha_{2}, \cdots \alpha_{r} ; x \\
\beta_{1}, \beta_{2} \cdots \beta_{s} ;
\end{array}\right]
$$

Where $\Delta(m ; n)$ stands for set of $\mathrm{m}$ parameters $\frac{n}{m}, \frac{n+1}{m}, \ldots \ldots \ldots \ldots . . \frac{n+m-1}{m}$

\section{GOULD AND HOPPER POLYNOMIAL}

It is denoted by the symbol $g_{n}^{m}(x, h)$ and is defined as

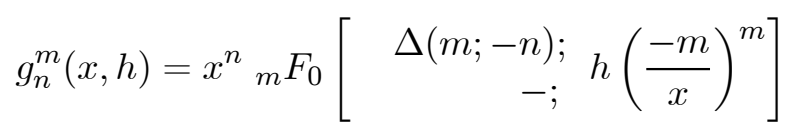

\section{LAHIRI POLYNOMIAL}

It is denoted by the symbol $H_{n, m, \nu}(x)$ and is defined as

$$
H_{n, m, \nu}(x)=(\nu x)^{n}{ }_{m} F_{0}\left[\begin{array}{r}
\Delta(m ;-n) ; \\
-;
\end{array}-\left(\frac{-m}{\nu x}\right)^{m}\right]
$$


It is denoted by the symbol $J_{n}^{(\alpha, \beta)}(x ; k)$ and is defined as (2.17)

$$
J_{n}^{(\alpha, \beta)}(x ; k)=\frac{(\alpha+1)_{k n}}{n !} k_{k+1} F_{n}\left[\begin{array}{r}
-n, \Delta(k ; \alpha+\beta+n+1) ; \\
\Delta(k ; \alpha+1) ;
\end{array}\left(\frac{1-x}{2}\right)^{k}\right]
$$

\section{SRIVASTAVA'S GENERALIZED HYPERGEOMETRIC POLY- NOMIAL}

It is denoted by the symbol $H_{n}^{(\alpha, \beta)}(x ; m)$ and is defined as

$$
H_{n}^{(\alpha, \beta)}(x ; m)=\left(\begin{array}{c}
\alpha+(\beta+1) n \\
n
\end{array}\right){ }_{p+m} F_{q+m}\left[\begin{array}{cc}
\Delta(m ;-n), & \left(a_{p}\right) ; \\
\Delta(m ; \alpha+\beta n+1),\left(b_{q}\right) ; & x
\end{array}\right]
$$

\section{Operational representations :}

If $D_{x} \equiv \frac{\partial}{\partial x}$ and $D_{y} \equiv \frac{\partial}{\partial y}$, M. A. Khan and A. K. Shukla [4] wrote the binomial expansion for $\left(D_{x}+D_{y}\right)^{n}$ as

$$
\left(D_{x}+D_{y}\right)^{n} \equiv \sum_{r=0}^{n}{ }^{n} C_{r} D_{x}^{n-r} D_{y}^{r}
$$

where ${ }^{n} C_{r}=\frac{n !}{r !(n-r) !}$. By writing the finite series on the right of (3.1) M.A. Khan and A.K. Shukla [4] wrote (3.1) also as

$$
\left(D_{x}+D_{y}\right)^{n} \equiv \sum_{r=0}^{n}{ }^{n} C_{r} D_{x}^{r} D_{y}^{n-r}
$$

If $\mathrm{F}(\mathrm{x}, \mathrm{y})$ is afunction of $\mathrm{x}$ and $\mathrm{y}$, they obtained the following from (3.1) and $(3.2)$

$$
\left(D_{x}+D_{y}\right)^{n} F(x, y) \equiv \sum_{r=0}^{n} \frac{(-n)_{r}(-1)^{r}}{r !} D_{x}^{n-r} D_{y}^{r} F(x, y)
$$




$$
\left(D_{x}+D_{y}\right)^{n} F(x, y) \equiv \sum_{r=0}^{n} \frac{(-n)_{r}(-1)^{r}}{r !} D_{x}^{r} D_{y}^{n-r} F(x, y)
$$

In particular , if $F(x, y)=f(x) g(y)$. M.A.Khan and A.K.Shukla [4] wrote (3.3) and (3.4) in the form

$$
\left(D_{x}+D_{y}\right)^{n} f(x) g(y) \equiv \sum_{r=0}^{n} \frac{(-n)_{r}(-1)^{r}}{r !} D_{x}^{n-r} f(x) D_{y}^{r} g(y)
$$

$\left(D_{x}+D_{y}\right)^{n} f(x) g(y) \equiv \sum_{r=0}^{n} \frac{(-n)_{r}(-1)^{r}}{r !} D_{x}^{r} f(x) D_{y}^{n-r} g(y)$

Now by taking special values of $f(x)$ and $g(y)$ in (3.5), we obtain the following partial differential operator representations of the polynomials given above :

$\left(D_{x}+D_{y}\right)^{n}\left\{x^{-s-1} y^{-1}\right\}=(-1)^{n} n ! x^{-s-1-n} y^{-1} g_{n}^{(s)}\left(\frac{x}{y}\right)$

$\left(1+D_{y} D_{z}\right)^{n}\left\{y^{-x} e^{\frac{-1}{x} z}\right\}=n ! y^{-x} e^{\frac{-1}{x} z} x^{-n} \varphi_{n}\left(\frac{y}{x}\right)$

$\left(1-D_{x}\right)^{n}\left\{x^{a-1+2 n}\right\}=n ! x^{a-1+2 n} R_{n}(a, x)$

$$
\begin{aligned}
& \left(D_{u}-D_{x} D_{y}\right)^{n}\left\{u^{-1} x^{\frac{n}{2}} y^{\frac{n}{2}-\frac{1}{2}}\right\}=(-1)^{n} n ! 2^{-n} u^{-n-1} x^{-\frac{n}{2}} y^{\frac{n}{2}-\frac{1}{2}} H_{n}\left(\sqrt{\frac{x y}{u}}\right) \\
& \left(D_{u} D_{v} D_{w}+D_{x} D_{y} D_{z}\right)^{n}\left\{u^{-1} v^{\gamma-1+n} w^{-\beta} x^{\frac{n}{2}} y^{\frac{n}{2}-\frac{1}{2}} z^{\beta-\gamma}\right\}
\end{aligned}
$$


$=(n !)^{2}(\gamma)_{n} 2^{-n} u^{-n-1} v^{\gamma-1} w^{\beta-n} x^{-\frac{n}{2}} y^{\frac{n}{2}-\frac{1}{2}} z^{\beta-\gamma} R_{n}\left(\beta ; \gamma ; \sqrt{\frac{x y z}{u v w}}\right)$

$\left(D_{u} D_{v} D_{w}+D_{x} D_{y} D_{z}\right)^{n}\left\{u^{-1} v^{-\alpha} w^{-\beta} x^{\frac{n}{2}} y^{\frac{n}{2}-\frac{1}{2}} z^{-1+\alpha+\beta+n}\right\}$

$=(-1)^{n}(n !)^{2}(\alpha+\beta)_{n} 2^{-n} u^{-n-1} v^{-\alpha-n} w^{-\beta-n} x^{\frac{n}{2}} y^{\frac{n}{2}-\frac{1}{2}} z^{\alpha+\beta+n-1} G_{n}\left(\alpha ; \beta ; \sqrt{\frac{x y z}{u v w}}\right)$

$\left(D_{u} D_{v} D_{w} D_{x}+D_{y} D_{z}\right)^{n}\left\{u^{-1} v^{-1} w^{\nu-1+n} x^{-\nu} y^{\frac{n}{2}} z^{\frac{n}{2}-\frac{1}{2}}\right\}$

$=(n !)^{2}(\nu)_{n} 2^{-n} u^{-1-\frac{1}{2}} v^{-n-1} w^{\nu-1} x^{-\nu-n} y^{\frac{n}{2}} z^{-\frac{n}{2}-\frac{1}{2}} R_{n, \nu}\left(\sqrt{\frac{x u v w}{y z}}\right)$

$\left(D_{x} D_{w_{1}} \cdots D_{w_{s}}-(-1)^{r+m} D_{y_{1}} \cdots D_{y_{m}} D_{z_{1}} \cdots D_{z_{r}}\right)^{n}$

$$
\times\left\{x^{-1} w_{1}^{\beta_{1}-1+n} \cdots w_{s}^{\beta_{s}-1+n} y_{1}^{\frac{n}{m}} \cdots y_{m}^{\frac{n-m+1}{m}} z_{1}^{-\alpha 1} \ldots z_{r}^{-\alpha_{r}}\right\}
$$

$=(n !)(-1)^{n}\left(\beta_{1}\right)_{n} \cdots\left(\beta_{s}\right)_{n} x^{-n-1} w_{1}^{\beta_{1}-1} \cdots w_{s}^{\beta_{s}-1} y_{1}^{\frac{n}{m}} \cdots y_{m}^{\frac{n-(m-n}{m}} z_{1}^{-\alpha_{1}} \cdots z_{r}^{-\alpha_{r}}$

$$
\times B_{n}^{m}\left[\alpha_{1} \cdots \alpha_{s} ; \beta_{1} \cdots \beta_{s} ; \frac{x w_{1} w_{2} \cdots w_{s}}{y_{1} y_{2} \cdots y_{m} z_{1} z_{2} \cdots z_{r}}\right]
$$

$\left(D_{x^{m}}+(-1)^{m} h\left(m^{m}\right) D_{y_{1}} \cdots D_{y_{m}}\right)^{n}\left\{\left(x^{-m}\right)^{-1} y_{1}^{\frac{n}{m}} \cdots y_{m}^{\frac{n-m+1}{m}}\right\}$

$=(-1)^{n} n ! x^{m n+m-n} y_{1}^{\frac{n}{m}} \cdots y_{m}^{\frac{n-m+1}{m}} g_{n}^{m}\left(x y_{1}^{\frac{1}{m}} \cdots \cdots y_{m}^{\frac{1}{m}}, h\right)$ 


$$
\left(D_{(v x)^{m}}+(-1)^{m}\left(-m^{m}\right) D_{y_{1}} \cdots D_{y_{m}}\right)^{n}\left\{\left[(v x)^{-m}\right]^{-1} y_{1}^{\frac{n}{m}} \cdots y_{m}^{\frac{n-m+1}{m}}\right\}
$$

$$
=(1)^{n} n ! v x^{m n+m-n} y_{1}^{\frac{n}{m}} \cdots y_{m}^{\frac{n m+1}{m}} H_{m, n, \nu}\left(x y_{1}^{\frac{1}{m}} \cdots \cdots y_{m}^{\frac{1}{m}}, h\right)
$$

$$
\left(D_{y_{1}} \cdots D_{y_{k}}-\left(\frac{-1}{2}\right)^{k} D_{z_{1}} \cdots D_{z_{k}}\right)^{n}
$$

$$
\times\left\{y_{1}^{\frac{\alpha+1}{k}-1+n} \cdots y_{k}^{\frac{\alpha+k}{k}-1+n} z_{1}^{-\frac{\alpha+\beta+n+1}{k}} \cdots z_{k}^{-\frac{\alpha+\beta+n+k}{k}}\right\}
$$

$$
=\frac{n !}{k^{k^{n}}} y_{1}^{\frac{\alpha+1}{k}-1} \cdots y_{k}^{\frac{\alpha+k}{k}-1} z_{1}^{-\frac{\alpha+\beta+n+1}{k}} \cdots z_{k}^{-\frac{\alpha+\beta+n+k}{k}} J_{n}^{(\alpha, \beta)}\left[1-2\left(\frac{y_{1} y_{2} \cdots \cdots y_{k}}{z_{1} z_{2} \cdots \cdots z_{k}}\right)^{\frac{1}{k}} ; k\right]
$$

$$
\left(D_{u} D_{v_{1}} \cdots D_{v_{m}} D_{w_{1}} \cdots D_{w_{q}}-(-1)^{m+r} D_{y_{1}} \cdots D_{y_{m}} D_{z_{1}} \cdots D_{z_{r}}\right)^{n}
$$

$$
\begin{gathered}
\times\left\{u^{-1} v_{1}^{\frac{\alpha+\beta n+1}{m}-1+n} \cdots v_{m}^{\frac{\alpha+\beta}{m}}-1+m w_{1}^{b_{1}-1+n} \cdots w_{q}^{b_{q}-1+n} y_{1}^{\frac{n}{m}} \cdots y_{m}^{\frac{n+m-1}{m}} z_{1}^{-a_{1}} \cdots z_{r}^{-a_{r}}\right\} \\
=(-1)^{n} n ! \frac{(\alpha+\beta n+1)_{m n}}{(1+\alpha+\beta n)_{m} m^{m n}}\left(b_{1}\right)_{n} \cdots\left(b_{q}\right)_{n} u^{-n-1} v_{1}^{\frac{\alpha+\beta n-m+1}{m}} \cdots v_{m}^{\frac{\alpha+\beta n)}{m}}
\end{gathered}
$$

$$
\times w_{1}^{b_{1}-1} \cdots w_{q}^{b_{q}-1} y_{1}^{\frac{n}{m}} \cdots y_{m}^{\frac{n+m-1}{m}} z_{1}^{-a_{1}} \cdots z_{r}^{-a_{r}} H_{n}^{(\alpha, \beta)}\left(\frac{u v_{1} v_{2} \cdots \cdots v_{m} w_{1} w_{2} \cdots \cdots w_{q}}{y_{1} y_{2} \cdots \cdots y_{m} z_{1} z_{2} \cdots \cdots z_{r}} ; m\right)
$$

Some proofs are given:

Proof of (3.7): 


$$
\begin{aligned}
& \left(D_{x}+D_{y}\right)^{n}\left\{x^{-s-1} y^{-1}\right\} \\
& =\sum_{r=0}^{n} \frac{(-n)_{r}(-1)^{r}}{r !} \frac{(1+s)_{n}(-1)^{n}}{(-s-n)_{r}} x^{-s-1-n+r}(1)_{r}(-1)^{r} y^{-1-r} \\
& =(-1)^{n} n ! x^{-s-1-n} y^{-1}\left(\begin{array}{l}
s+n \\
n
\end{array}\right){ }_{2} F_{1}\left[\begin{array}{cc}
-n, 1 ; & x \\
-s-n ; & y
\end{array}\right] \\
& \quad=(-1)^{n} n ! x^{-s-1-n} y^{-1} g_{n}^{(s)}\left(\frac{x}{y}\right)
\end{aligned}
$$

\section{Proof of (3.8):}

$$
\begin{aligned}
& \left(1+D_{y} D_{z}\right)^{n}\left\{y^{-x} e^{\frac{-1}{x} z}\right\} \\
& =\sum_{r=0}^{n} \frac{(-n)_{r}(-1)^{r}}{r !} D_{y}^{r}\left\{y^{-x}\right\} D_{z}^{r}\left\{e^{\frac{-1}{x} z}\right\} \\
& =n ! y^{-x} e^{\frac{-1}{x} z} x^{-n} \frac{x^{n}}{n !}{ }_{2} F_{0}\left[\begin{array}{rr}
-n, & x \\
& ; \frac{-1}{x y}
\end{array}\right] . \\
& =n ! y^{-x} e^{\frac{-1}{x} z} x^{-n} \varphi_{n}\left(\frac{y}{x}\right)
\end{aligned}
$$

\section{Proof of (3.9):}

$$
\begin{aligned}
& \left(1-D_{x}\right)^{n}\left\{x^{a-1+2 n}\right\} \\
& =\sum_{r=0}^{n} \frac{(-n)_{r}(-1)^{n-r}}{r !} \frac{(a+n)_{n}}{(a+n)_{r}} x^{a+n-1+r} \quad \text { Using (3.6) } \\
& =n ! \frac{(a)_{2} n}{(a)_{n}} x^{a-1+n}{ }_{1} F_{1}\left[\begin{array}{c}
-n ; \\
a+n ;
\end{array}\right] .
\end{aligned}
$$




$$
=n ! x^{a-1+n} R_{n}(a, x)
$$

\section{Proof of (3.10):}

$$
\begin{aligned}
& \left(D_{u}-D_{x} D_{y}\right)^{n}\left\{u^{-1} x^{\frac{n}{2}} y^{\frac{n}{2}-\frac{1}{2}}\right\} \\
& =\sum_{r=0}^{n} \frac{(-n)_{r}}{r !} \frac{(-1)^{n} n !}{(-n)_{r}} u^{-n-1+r}\left(\frac{-n}{2}\right)_{r}(-1)^{r} x^{\frac{n}{2}-r}\left(\frac{-n}{2}+\frac{1}{2}\right)_{r}(-1)^{r} y^{\frac{n}{2}-\frac{1}{2}-r} \\
& =(-1)^{n} n ! u^{-n-1} x^{\frac{n}{2}} y^{\frac{n}{2}-\frac{1}{2}}{ }_{2} F_{0}\left[\begin{array}{l}
-\frac{n}{2}, \frac{-n}{2}+\frac{1}{2} ; \frac{u}{x y} \\
;
\end{array}\right] \\
& =(-1)^{n} n ! 2^{-n} u^{-n-1} x^{-\frac{n}{2}} y^{\frac{n}{2}-\frac{1}{2}} H_{n}\left(\sqrt{\frac{x y}{u}}\right)
\end{aligned}
$$

\section{Proof of (3.11):}

$$
\begin{aligned}
& \left(D_{u} D_{v} D_{w}+D_{x} D_{y} D_{z}\right)^{n}\left\{u^{-1} v^{\gamma-1+n} w^{-\beta} x^{\frac{n}{2}} y^{\frac{n}{2}-\frac{1}{2}} z^{\beta-\gamma}\right\} \\
& =\sum_{r=0}^{n} \frac{(-n)_{r}(-1)^{r}}{r !} \frac{(-1)^{n} n !}{(-n)_{r}} u^{-n-1+r} \frac{(\gamma)_{n}}{(\gamma)_{r}} v^{\gamma-1+r} \frac{(\beta)_{n}(-1)^{n}}{(1-\beta-n)_{r}} \\
& \times w^{-\beta-n+r}\left(\frac{-n}{2}\right)_{r}(-1)^{r} x^{\frac{n}{2}-r}\left(\frac{-n}{2}+\frac{1}{2}\right)_{r}(-1)^{r} y^{\frac{n}{2}-\frac{1}{2}-r}(\gamma-\beta)_{r}(-1)^{r} z^{\beta-\gamma-r} \\
& =n !(\beta)_{n}(\gamma)_{n} u^{-n-1} v^{\gamma-1} w^{-\beta-n} x^{\frac{n}{2}} y^{\frac{n}{2}-\frac{1}{2}} z^{\beta-\gamma} \\
& \sum_{r=0}^{n} \frac{\left(\frac{-n}{2}\right)_{r}\left(\frac{-n}{2}+\frac{1}{2}\right)_{r}(\gamma-\beta)_{r}}{r !(\gamma)_{r}(1-\beta-n)_{r}}\left(\frac{u v w}{x y z}\right)^{r} \\
& =\frac{(n !)^{2}(\gamma)_{n}}{2^{n}} u^{-n-1} v^{\gamma-1} w^{\beta-n} x^{-\frac{n}{2}} y^{\frac{n}{2}-\frac{1}{2}} z^{\beta-\gamma} \frac{(\beta)_{n}(2 x)^{n}}{n !} 3 \\
& F_{2}\left[\begin{array}{l}
-\frac{n}{2}, \frac{-n}{2}+\frac{1}{2}, \gamma-\beta ; \frac{u u w}{x y z} \\
\gamma, 1-\beta-n ;
\end{array}\right] \\
& =(n !)^{2}(\gamma)_{n} 2^{-n} u^{-n-1} v^{\gamma-1} w^{\beta-n} x^{-\frac{n}{2}} y^{\frac{n}{2}-\frac{1}{2}} z^{\beta-\gamma} R_{n}\left(\beta ; \gamma ; \sqrt{\frac{x y z}{u v w}}\right)
\end{aligned}
$$




\section{Proof of (3.16):}

$$
\begin{aligned}
& \left(D_{(v x)^{m}}+(-1)^{m}\left(-m^{m}\right) D_{y_{1}} \cdots D_{y_{m}}\right)^{n}\left\{\left[(v x)^{-m}\right]^{-1} y_{1}^{\frac{n}{m}} \cdots y_{m}^{\frac{n-m+1}{m}}\right\} \\
& =\sum_{r=0}^{n} \frac{(-n)_{r}(-1)^{r}}{r !} \frac{(-n)_{n}}{(-n)_{r}}\left[(v x)^{-m}\right]^{-n-1+r}\left[(-1)^{m}\right]^{r}(-m)^{r} \\
& \quad \times\left(\frac{-n}{m}\right)_{r}(-1)^{r} y_{1}^{\frac{n}{m}-r} \ldots\left(\frac{-n+m-1}{m}\right)_{r}(-1)^{r} y_{m}^{\frac{n-m+1}{m}-r} \\
& =(-1)^{n} n !(v x)^{m n+m} y_{1}^{\frac{n}{m}} \cdots \cdots y_{m}^{\frac{n-m+1}{m}}{ }_{m} F_{0}\left[\Delta(m, n) ;-\left(\frac{-m}{x y_{1}^{\frac{1}{m}} \cdots \cdots y_{m}^{\frac{1}{m}}}\right)^{m}\right] \\
& =(1)^{n} n ! v x^{m n+m-n} y_{1}^{\frac{n}{m}} \cdots y_{m}^{\frac{n m+1}{m}} H_{m, n, \nu}\left(x y_{1}^{\frac{1}{m}} \cdots \cdots y_{m}^{\frac{1}{m}}, h\right)
\end{aligned}
$$

\section{References}

[1] Andrews, G. E., Askey, R. and Roy, R.: Special functions. Cambridge University Press, (1999).

[2] Bedient,P. E.: Polynomials related to Appell functions of two variables. Michigan thesis, (1958).

[3] Chihara, T. S.: An introdution to Orthogonal Polynomials. Gordon and Breach, New York, (1978).

[4] Khan, M. A. and Shukla, A. K.: On Binomial and Trinomial operator representations of certain Polynomials. Italian Journal of Pure and Applied Mathematics- N. 25, pp. 103-110, (2009).

[5] Khan, M. A. and Nisar K. S. : On bilinear operator representations of Laguerre and Jacobi polynomials. International transactions in Applied Sciences, 1, pp. 521-526, (2009). 
[6] Khan, M. A. and Nisar K. S.: Bilinear and Bilateral summation formulae of certain polynomials in the form of operator representations. Accepted for publication in Thai J. Mathematics, (2010).

[7] Khan, M. A. and Nisar K. S.: On operator representations of various polynomials using the operator of W.A. Al-Salam. Int. J. Computational and Applied Math., Volume 5, N 3, pp. 393-405, (2010).

[8] Khan, M. A. and Nisar K. S.: Operationalrepresentations of Bilinear and Bilateral Generating formulae for polynomials. Mathematical Sciences and Engg Applications, 3, pp. 1-19, (2010).

[9] Rainville, E. D.: Special Functions, MacMillan, New York (1960), Reprinted by Chelsea Publishing Company, Bronx, New York (1971).

[10] Srivastava, H. M. and Manocha, H. L. : A Treatise on Generating Functions, Ellis Horwood Limited, Chichester, (1984).

\section{Mumtaz Ahmad Khan}

Department of Applied Mathematics,

Faculty of Engineering and Technology,

Aligarh Muslim University,

Aligarh - 202002, U. P.,

India

e-mail: mumtaz_ahmad_khan_2008@yahoo.com

and

\section{Nisar K. S.}

Department of Applied Mathematics,

Faculty of Engineering and Technology,

Aligarh Muslim University,

Aligarh - 202002, U. P.,

India

e-mail: ksnisar1@gmail.com 